\title{
The effect of gold nanoparticles on the impedance of microcapsules visualized by scanning photo-induced impedance microscopy
}

Wang, J; Campos, I; Wu, F; Zhu, J; Sukhorukov, GB; Palma, M; Watkinson, M; Krause, S

(C) 2016 Elsevier Ltd.

For additional information about this publication click this link.

http://qmro.qmul.ac.uk/xmlui/handle/123456789/12317

Information about this research object was correct at the time of download; we occasionally make corrections to records, please therefore check the published record when citing. For more information contact scholarlycommunications@qmul.ac.uk 
The effect of gold nanoparticles on the impedance of microcapsules visualized by scanning photo-induced impedance microscopy

Jian Wang ${ }^{1}$, Inmaculada Campos ${ }^{1}$, Fan $\mathrm{Wu}^{1}$, Jingyuan $\mathrm{Zhu}^{2}$, Gleb B. Sukhorukov ${ }^{1}$, Matteo Palma², Michael Watkinson ${ }^{2}$, Steffi Krause ${ }^{1 *}$

${ }^{1}$ School of Engineering and Materials Science, Queen Mary University of London, Mile End Road, London E1 4NS, UK

${ }^{2}$ School of Biological and Chemical Sciences, Queen Mary University of London, Mile End Road, London E1 4NS, UK

${ }^{*}$ Corresponding authors: s.krause@gmul.ac.uk, Telephone +44 (0)2078823747 and m.watkinson@qmul.ac.uk, Telephone +44 (0)2078823263

\begin{abstract}
:
Polyelectrolyte microcapsules have attracted great interest in drug delivery applications, and microcapsules modified with gold nanoparticles have been used in this way with triggered release when a laser can be used to remotely open shells through light-induced local heating. The electrical impedance of unmodified microcapsules has been studied due to its implications for their permeability, however, the impedance of functionalised microcapsules has not yet been investigated. Herein, the impedance of microcapsules modified with gold nanoparticles was studied for the first time. It was shown that the modification of microcapsules with gold nanoparticles leads to a much greater impedance than would be expected from the increase in the thickness caused by the presence of a layer of gold nanoparticles alone. The impedance of gold nanoparticle modified capsules was measured using scanning photo-induced impedance microscopy
\end{abstract}


(SPIM), which is based on photocurrent measurements at an electrolyte-insulatorsemiconductor (EIS) field-effect structure. High resolution and good sensitivity were achieved using a two-photon effect for charge carrier excitation and organic monolayer modified silicon on sapphire (SOS) as the SPIM substrate. SPIM allowed impedance imaging of collapsed microcapsules with unprecedented detail. SPIM images of capsules labelled with gold nanoparticles (AuNPs) showed a good agreement with the corresponding optical images, including the creases resulting from the collapse of the hollow shells. The significant increase in impedance caused by the impregnation with AuNPs was also verified by conductive Atomic Force Microscopy (C-AFM) measurements in the dry state.

Keywords: Impedance, polyelectrolyte microcapsules, Scanning Photo-induced Impedance Microscopy (SPIM), Light-Addressable Potentiometric Sensors (LAPS), gold nanoparticles.

\section{Introduction}

Polymeric multilayer capsules (PMLCs) assembled by layer-by-layer (LBL) approach on a sacrificial template continue to receive considerable attention because of their wide ranging applications in drug delivery, sensing, microreactors and smart materials [1-3]. Whilst the LBL assembly technique allows the design and modification of hollow microcapsules in a controllable way, the impregnation of the capsule shell with gold nanoparticles (AuNPs) is undoubtedly one of the most attractive ways to tailor their functionality [4-7]. For example, laser-induced remote drug release is based on localized heating due to surface plasmon resonance absorption by the nanoparticles [8-10]. Extensive research has also been carried out into the light sensitivity of AuNP modified PMLCs, and force deformation 
experiments have shown an improvement in the shell stiffness due to doping with AuNPs [11]. Moreover, a higher bending stiffness, elastic modulus and robustness have also been reported for free standing poly(allyl amine hydrochloride) (PAH)/ poly(sodium 4-styrene sulfonate) (PSS) membranes with a central layer containing gold nanoparticles [12]. It has been reported that the ionic permeability and the integrity of the PMLC wall are closely related to their electrical impedance [13],[14]. Electrorotation [13] and a microfluidic single particle impedance chip[14] have both been used to measure the impedance of PAH/PSS microcapsules reporting dielectric constants of 60 and 50 indicating a high water content of the capsule wall. However, the effect of AuNP doping on the electrochemical properties has not yet been studied. In this work, we investigated the effects of AuNPs on the impedance of the hollow microcapsules using scanning photo-induced impedance microscopy (SPIM) for the first time as this has implications for the permeability and stability of these capsules in physiological media and therefore their suitability for drug delivery. The sensitivity and resolution of SPIM has recently been improved significantly [15, 16] and is therefore an attractive tool for the investigation of the electrical impedance of PMLCs. SPIM is based on photocurrent measurement at electrolyte-insulatorsilicon (EIS) field-effect structures and can provide a two-dimensional impedance image of the sample surface [17-19]. A bias voltage is applied between the semiconductor and a reference electrode immersed in the electrolyte to obtain an inversion layer, and a modulated light beam focused into the semiconductor scans across the substrate to generate electron/hole pairs. The separation of electron/hole pairs in the space charge layer results in ac photocurrents relating to the local impedance. High-quality impedance images of polymer films were obtained using SPIM, and a good agreement with the spectra measured with classical ac- 
impedance spectroscopy was confirmed [18]. By measuring local photocurrents while biasing the EIS structure towards depletion, local surface potentials could also be recorded - a technique termed light-addressable potentiometric sensors (LAPS) [20-22].

Submicrometre resolution for SPIM and LAPS has been achieved using silicon on sapphire (SOS, $0.5 \mu \mathrm{m}$ silicon, $475 \mu \mathrm{m}$ sapphire) as the semiconductor substrate and photoexcitation of charge carriers using a two photon-effect in the thin silicon layer by employing a femtosecond laser $(1250 \mathrm{~nm})$ with photon energy smaller than the bandgap of silicon [17]. By replacing the traditional insulator with a selfassembled organic monolayer that was bound to hydrogen-terminated silicon on the SOS substrate, we have significantly increased the sensitivity of SPIM and the measurement accuracy of LAPS recently $[15,16]$. The use of a self-assembled organic monolayer of 1,8-nonadiyne as the insulator has the advantage that different surface functionalities can be subsequently introduced using a simple $\mathrm{Cu}(\mathrm{I})$ catalysed "click" cycloaddition between the alkyne of the monolayer and an azide [15, 16]. It was therefore decided to perform high-resolution SPIM measurements of PMLCs based on the well-established monolayer modified SOS substrates and the femtosecond laser in order to investigate the effects of AuNPs on their impedance.

\section{Experimental}

\subsection{Materials}

Monodisperse melamine formaldehyde (MF) microparticles ( 10 $\mu \mathrm{m}$, dispersed in water) were purchased from Microparticles $\mathrm{GmbH}$, Germany. A suspension of gold nanoparticles stabilised with citrate $(\sim 15 \mathrm{~nm}$, dispersed in water) were prepared following a procedure described elsewhere [23]. 
Silicon-on-sapphire (SOS) with a $1 \mu \mathrm{m}$ thick silicon (100) layer (boron doped, $0.1 \Omega \mathrm{cm}$ ) on a $475 \mu \mathrm{m}$ thick sapphire substrate was purchased from Monocrystal, Russia. Double polished silicon (100) (boron doped, 10-30 $\Omega \mathrm{cm}$ ) was purchased from Si-MAT, Germany. All chemicals and reagents, unless otherwise noted, were purchased from Sigma-Aldrich and were used as received. Dichloromethane (DCM) used for cleaning was redistilled prior to use. 1,8-Nonadiyne (98\%) was redistilled from sodium borohydride $(99+\%)$ and stored under argon as reported [24, 25]. Argon was dried and purified through an oxygen/moisture trap (Agilent Technologies, USA).

\subsection{Microcapsule preparation and characterisation}

To study the effect of AuNPs on the impedance of capsules, hollow capsules with AuNPs $(\sim 15 \mathrm{~nm})(\text { PSS/PAH) })_{2}$-AuNPs-(PSS/PAH) $)_{2}$ and without AuNPs $(P S S / P A H)_{4}$ were prepared using MF microparticles as templates following a previously established procedure [26] (see ESI for further details).

The distribution of AuNPs in the shell of microcapsules was observed by a transmission electron microscope (TEM, JEOL 2010) operated at $200 \mathrm{kV}$. The diluted capsule suspension was deposited on a carbon-coated copper grid, and airdried for $2 \mathrm{~h}$. Then, the copper grid was installed in a sample holder and placed into the vacuum chamber of the TEM for characterisation.

Zeta potentials of hollow capsules containing AuNPs were measured with a Malvern Nano ZS zetasizer (Malvern Instruments Ltd, UK). For measurements, a small volume of sample solution was transferred to a transparent cuvette, diluted with pure water and placed in the thermostatted cavity. Fifteen measurements were taken on each sample and averaged. Atomic Force Microscopy (AFM, Dimension Icon, Bruker, US) in PeakForce TUNA mode was used to image the morphology and conductivity 
distribution of collapsed microcapsules. A Bruker PeakForce TUNA tip (Au coating, spring constant of $0.4 \mathrm{~N} / \mathrm{m}$ ) was used for scanning and a dc bias voltage at $1 \mathrm{~V}$ was applied between the AFM tip and the sample substrate.

\subsection{SPIM substrate preparation and characterisation}

SOS wafers and double polished silicon wafers were cut into $7 \mathrm{~mm} \times 7 \mathrm{~mm}$ pieces. In order to form the ohmic contact for SPIM/LAPS measurements, $30 \mathrm{~nm} \mathrm{Cr}$ and $150 \mathrm{~nm}$ Au were thermally evaporated onto one corner of the chip and subsequently heated to $300{ }^{\circ} \mathrm{C}$ for $5 \mathrm{~min}$ as previously reported [15]. The substrate was cleaned in a hot piranha solution (3:1 $\mathrm{H}_{2} \mathrm{SO}_{4}(96 \%) / \mathrm{H}_{2} \mathrm{O}_{2}(30 \%)$, caution: highly corrosive) at $100{ }^{\circ} \mathrm{C}$ for $30 \mathrm{~min}$ and then rinsed copiously with ultrapure (Milli-Q) water. The assembly of the 1,8-nonadiyne monolayer followed the procedure reported by Ciampi et al. [24, 25]. The cleaned SOS or silicon sample was transferred to a $2.5 \%$ $\mathrm{HF}$ solution and chemically etched for $90 \mathrm{~s}$ to obtain the $\mathrm{H}$-terminated surface (caution: HF is highly corrosive). During the cleaning and etching time, the redistilled 1,8-nonadiyne was transferred into a Schlenk tube and was degassed by freezepump-thaw cycles until no gas bubbles evolved from the solution. Then, the freshly prepared $\mathrm{H}$-SOS or $\mathrm{H}$-Si sample was transferred into the degassed 1,8-nonadiyne and left for $3 \mathrm{~h}$ at $165^{\circ} \mathrm{C}$ under an argon stream. After cooling to room temperature, the functionalized surface was then rinsed with copious amounts of redistilled DCM and blown dry with nitrogen. Figure 1 shows the process of monolayer formation and the subsequent copper catalysed azide alkyne cycloadditon (CuAAC) or "click" functionalisation on SOS which was carried out as previously reported [16]. The same surface modification was performed on silicon substrates for ellipsometry. The alkyne-terminated surface was further functionalized through a typical "click" 
procedure [16]. The substrate was transferred into a vessel containing the "click" solution of the 4-azidobenzoic acid (1 ml, $15 \mathrm{mM}$, ethanol/water 2:1), copper(II) sulfate pentahydrate $(1.1 \mathrm{~mol} \%$ relative to the azide $)$, sodium ascorbate $(10 \mathrm{~mol} \%$ relative to the azide) and TMEDA $(0.45 \mathrm{mM})$. The reaction was carried out in the dark at room temperature for $24 \mathrm{~h}$. The unreacted reagents were removed by rinsing the substrate consecutively with copious amounts of ethanol, water and ethanol. Then, the sample was placed in a $0.5 \mathrm{M}$ hydrochloric acid solution for $2 \mathrm{~min}$ to remove the residual copper [27]. Finally, it was washed with copious amounts of water and ethanol. For SPIM measurements, to attach the microcapsules to the surface, a diluted capsule suspension was deposited on the SPIM substrate, and airdried for $2 \mathrm{~h}$ analogous to the preparation of the TEM sample.

The water wettability of monolayer modified SOS samples was measured using a Drop Shape Analysis System (Krüss DSA100, Germany). $1 \mu \mathrm{L}$ of ultrapure water was carefully deposited onto the surface and three spots were measured on each sample and averaged.

An alpha-SE® Spectroscopic Ellipsometer and CompleteEASE software (J.A. Woollam Co. Inc., USA) for data collection and analysis were used to measure the thickness of monolayers on silicon surfaces. The wavelength range for measurements was $380-900 \mathrm{~nm}$ and the angle of incidence was $70^{\circ}$. X-ray photoelectron spectroscopy (XPS) experiments were performed on modified SOS surfaces at the nanoLAB of Newcastle University, UK, using a Kratos Axis Nova spectrometer with CasaXPS software. Survey scans were carried out over a $1100 \sim 0 \mathrm{eV}$ range with a $1.0 \mathrm{eV}$ step size, a $100 \mathrm{~ms}$ dwell time, and an analyzer pass energy of $100 \mathrm{eV}$. High-resolution scans were run with a $0.1 \mathrm{eV}$ step size, a dwell time of $100 \mathrm{~ms}$, and the analyser pass energy set to $20 \mathrm{eV}$. The scan regions were 
Si 2p (97-107 eV), C 1s (278-294 eV), N 1s (392-408 eV), O 1s (526-542 eV) and Cu 2p3/2 (926-938 eV). The CasaXPS software (nanoLAB of Newcastle University, UK) was used to analyse the spectra. The background was subtracted using the Shirley routine, and then the spectra were fitted with a line shape following the mixed Gaussian-Lorentzian (GL) functions.

\subsection{SPIM measurements}

Figure 2a shows the principle of the SPIM system based on a sandwich structure containing a p-SOS substrate with an organic monolayer and an electrolyte solution (10 mM phosphate buffer solution pH 7.4 containing $137 \mathrm{mM} \mathrm{NaCl}$ and $2.7 \mathrm{mM} \mathrm{KCl}$ ). A bias voltage was applied between the silicon and an $\mathrm{Ag} / \mathrm{AgCl}$ electrode immersed in the electrolyte. The femtosecond laser $(1250 \mathrm{~nm}-\mathrm{a}$ wavelength that is not absorbed by the AuNPs) was chopped at $1 \mathrm{kHz}$ and focused on the $1 \mu \mathrm{m}$ thick silicon layer. The sample holder was mounted on an XYZ positioning system, which allowed the laser to be focused and an image to be built by moving the sample with respect to the laser beam. AC photocurrents were recorded using a lock-in amplifier. The detailed set-up is shown in Figure S2 and was previously shown to have a resolution of $0.8 \mu \mathrm{m}$ [17]. A schematic of the photocurrent curves recorded is shown in Figure 2b.

\section{Results and discussion}

\subsection{Microcapsule characterisation}

To study the effect of AuNPs on the impedance of capsules, hollow capsules with AuNPs $(\sim 15 \mathrm{~nm})(P S S / P A H)_{2}-A u N P s-(P S S / P A H)_{2}$ and without AuNPs $(P S S / P A H)_{4}$ were prepared. Figure 3 shows the TEM images of collapsed capsules $(P S S / P A H)_{2}-$ 
AuNPs-(PSS/PAH $)_{2}$. It can be seen that the size of the microcapsules is consistent with the size of the MF core used as the template for empty capsule fabrication. The hollow nature of the microcapsules was confirmed by the completely collapsed morphology, which is characterised by an uneven thickness caused by folds and creases. Such behaviour of capsules is common due to the thin shell of about $20 \mathrm{~nm}$, which is weak enough to collapse upon drying $[4,6]$. Gold nanoparticles $(\sim 15 \mathrm{~nm})$ are clearly seen in the TEM image due to the high contrast with the polymeric materials of the capsule. The AuNPs were uniformly distributed over the whole capsule surface without aggregation and the average zeta potential was measured to be $+12 \mathrm{mV}$ for $(P S S / P A H)_{2}-A u N P s-(P S S / P A H)_{2}$ due to the positively charged PAH outer layer.

\subsection{SPIM substrate characterisation}

Advancing water contact angle, ellipsometry and XPS were used to demonstrate the presence and functionalisation of monolayers on the SOS and silicon substrates. Before "click" modification, the contact angle for the 1,8-nonadiyne modified surface was $90 \pm 1^{\circ}$, indicative of a hydrophobic surface due to the alkyne-terminated monolayer $[24,25,28]$. The ellipsometric thickness measured on the silicon substrate was $\sim 10 \AA$, indicating a tilt angle of $\sim 30^{\circ}$ between the axis of the monolayer molecules and the surface normal. After the "click" reaction with 4azidobenzoic acid, the substrate became hydrophilic with a water contact angle of $\sim 58^{\circ}$, which is comparable with the reported value [29], and the monolayer thickness increased to $\sim 18 \AA$, both of which are consistent with the successful surface modification. 
Since all XPS results acquired on the 1,8-nonadiyne monolayer surface agree with our previous report [16], they will not be discussed in detail here (see Figure S3). However, after "click" modification, the XPS survey scan (Figure 4a) showed the presence of nitrogen species at $\sim 401 \mathrm{eV}$ associated with formation of the "click" triazole. The absence of $\mathrm{Cu} 2 \mathrm{p} 3 / 2$ emission at $\sim 933 \mathrm{eV}$ indicated that there is no residual copper on the surface. A high-resolution scan of the $\mathrm{N}$ 1s region (Figure $4 \mathrm{~b}$ ) was fitted to two peaks at $400.5 \mathrm{eV}$ and $402.2 \mathrm{eV}$, which were assigned to $\mathrm{N}-\underline{\mathrm{N}}=\underline{\mathrm{N}}$ and $\underline{N}-\mathrm{N}=\mathrm{N}$, respectively $[27,30]$ and the ratio of the integrated areas $(\sim 2.2: 1)$ is comparable to the expected stoichiometric ratio (2:1). The narrow scan of the $C 1 \mathrm{~s}$ region (Figure 4c) was fitted with three peaks assigned to $\mathrm{C}-\mathrm{C}(285.1 \mathrm{eV}), \underline{\mathrm{C}}-\mathrm{N} /-\mathrm{O}$ $(286.7 \mathrm{eV})$ and $\underline{\mathrm{C}}=\mathrm{O}(289.5 \mathrm{eV}) \cdot[27,29,31]$ Encouragingly, no detectable photoemission of $\mathrm{SiO}_{x}$ species was observed in the $\mathrm{Si} 2 \mathrm{p}$ high-resolution spectrum, indicative of the high quality of the modified surface and monolayer integrity (Figure $4 d)$.

\subsection{SPIM imaging of microcapsules labelled with AuNPS}

SPIM measures local impedance changes by recording the maximum photocurrent in inversion, while LAPS is sensitive to potential changes, which appear as a shift of the depletion region of the photocurrent voltage $(I-V)$ curve along the voltage axis (see Figure 2b). Before photocurrent measurements, hollow capsules ((PSS/PAH) ${ }_{4}$ and $(\mathrm{PSS} / \mathrm{PAH})_{2}$-AuNPs-(PSS/PAH $)_{2}$ ) with a positively charged outer layer (PAH) were placed and collapsed on the SPIM substrate to achieve a good contact of the shells with the negatively charged substrate (-COO-).

Figure 5a presents the SPIM image of (PSS/PAH) ${ }_{2}-A u N P s-(P S S / P A H)_{2}$ measured at $0.9 \mathrm{~V}(\mathrm{vs} . \mathrm{Ag} / \mathrm{AgCl})$, while Figure $5 \mathrm{~b}$ shows the corresponding optical image. The 
SPIM image shows excellent agreement with the optical image of the microcapsules. The photocurrents on areas with attached capsules were lower than those on background monolayer surfaces, indicating an increase of the local impedance, as expected. The SPIM image also shows a remarkable level of detail as creases and overlapping areas of the collapsed microcapsules can clearly be distinguished as lines with relatively low photocurrents, which match the dark areas in the optical image. A higher resolution image of a single capsule is shown in Figure 5c.

The corresponding I-V curves measured on capsules (points " 1 " to "6" marked in Figure 5a) and off capsules (randomly selected on the background surface) are presented in Figure $5 e$. An average drop of $\sim 15 \%$ of the maximum photocurrents was observed at points "1", "2" and "3". However, when measured at the lines with lower photocurrents visible in the image, more significant drops ( $31 \%$ at points " 4 " and " 5 ", $\sim 41 \%$ at point " 6 ") of the maximum photocurrents were obtained (also see Figure S4). This is to be expected as these points represent thicker layers of the collapsed microcapsules. To obtain a LAPS image that is not affected by changes in the maximum photocurrent, a phase angle image was recorded at $0.6 \mathrm{~V}$ (Figure $5 \mathrm{~d}$ ) as described in [32]. The phase angle is lower in areas where the capsule is attached indicating a negative shift of the photocurrent-voltage curve due to the positive surface charge of the capsules. The phase angle was lower in areas where a lower photocurrent was measured (Figure $5 d$ ), i.e. in the areas where creases and folds are present, the surface charge of the capsules appears greater. This could be because folds and creases are likely to have a greater surface area and therefore more amino groups. Normalised $I-V$ curves on uncoated areas of the samples and points "1" to "6" on the capsules confirmed that there is a correlation between the negative voltage shift and the local thickness of the capsule. On average $I-V$ curves 
showed a negative shift of $(15 \pm 4) \mathrm{mV}$ (see Figure $5 f$ ) due to the PAH outer layer of the capsules, which is entirely consistent with the positive zeta potential of $12 \mathrm{mV}$. When SPIM is operated with a fixed light source and the double layer and surface state capacitances are negligible, the detectable photocurrent I can be modelled with an equivalent circuit (Figure 6) and written with the simplified equation [33]:

$I=I_{p} \frac{C_{\text {insulator }}}{C_{\text {insulator }}+C_{d}}(1)$

Where $I_{p}$ is the photon-induced current; $C_{\text {insulator }}$ and $C_{d}$ are the capacitances of the insulator and the depletion layer in the silicon. The behaviour of a LAPS substrate can be approximated with a pure capacitance in photocurrent measurements. The phase angle between photocurrent and light modulation measured in inversion at voltages $>0.9 \mathrm{~V}$ does not change in the area where capsules are attached. Hence it can be assumed that the capsule surface within the laser focus can be considered as a homogeneous dielectric with defined thickness and dielectric constant, which can also be modelled as a capacitance. The expression for the ratio of maximum photocurrents without $\left(I_{\max }\right)$ and with microcapsules $\left(I_{\max }(\right.$ capsule) $)$ can be simplified as below [33]:

$\frac{I_{\max }}{I_{\max (\text { capsule })}}=\frac{C_{m}}{C_{m}+C_{d}} \frac{C_{m-c}+C_{d}}{C_{m-c}}$

$C_{m-c}^{-1}=C_{m}^{-1}+C_{c}^{-1}$

Where $C_{m}$ and $C_{c}$ are capacitances of the organic monolayer and collapsed microcapsule, respectively. Since $C_{m}$ and $C_{d}$ can be approximated to be $1.7 \mu \mathrm{F} \mathrm{cm}^{-2}$ and $0.27 \mu \mathrm{F} \mathrm{cm}^{-2}$,[15] $C_{c}$ was calculated to be $\sim 1.4 \mu \mathrm{F} \mathrm{cm}^{-2}$ in the fold-free region (points "1", "2" and " 3 "), $0.57 \mu \mathrm{F} \mathrm{cm}^{-2}$ in the folded region (points "4" and "5") and $0.39 \mu \mathrm{F} \mathrm{cm}^{-2}$ in another folded region (point "6"). In parallel, the capsule shell 
thickness was measured by AFM. Figure 7a presents the topographical image of a capsule with AuNPs dried on a monolayer modified silicon surface. A typical grainlike surface structure was observed, which is consistent with previous studies [34]. The wall thickness of the capsule, which included two layers of the collapsed shell, was determined from the height difference between the silicon background ("i") and a fold-free region ("ii”). From the height profile along the rectangle (Figure 7b), the wall thickness was evaluated to be $\sim 25 \mathrm{~nm}$. From the thickness and the capacitance, a dielectric constant of 39 was calculated. This is considerably lower than the values of 50 and 60 reported for pure PAH/PSS capsules previously and is assumed to be due to the significantly reduced water content of the capsule shell when modified with AuNPs. Converting the photocurrent changes into thickness values indicates that points "4" and " 5 " in Figure 5 are on folded regions with a thickness of $61 \mathrm{~nm}$ and point " 6 " is on a folded region with a thickness of $89 \mathrm{~nm}$. These results demonstrate that the lines with lower photocurrent shown in Figure 6a do indeed correspond to the creases in the collapsed capsules.

\subsection{The effect of gold nanoparticles on the impedance of microcapsules}

SPIM and AFM measurements have also been performed on microcapsules without labelling $\left((P S S / P A H)_{4}\right)$ using the same measurement systems. The thickness in nonfolded regions was determined to be $18 \mathrm{~nm}$ via AFM topographical imaging (Figure 7d) indicating that labelling with AuNPs caused an increase of $7 \mathrm{~nm}$ in the thickness of two overlapping shells. In SPIM images, no photocurrent changes were observed in the presence of unlabelled microcapsules, which was unexpected considering that the shell thickness of the unmodified capsules is within the same order of magnitude as that of those modified with AuNP's. However, when estimating the capacitance of 
the unmodified shells using the dielectric constant of 60 reported previously [13], a value of $3.0 \mu \mathrm{F} \mathrm{cm}-2$ is obtained, which means that from Equation 2 a photocurrent drop of $8 \%$ should have been observed with SPIM. However, because of the inhomogeneous nature of the silicon on the SOS substrate [15], the maximum photocurrents in the background area always fluctuate in a range of about $\pm 10 \%$ as can be clearly seen in the off-capsule image of Figure 5a, which could therefore mask such small photocurrent changes caused by the attachment of microcapsules. The large capacitance of the polyelectrolyte multilayers is probably due to their porous structure [35] leading to a large water content, which has previously been estimated to be up to $50 \%$ of the layer volume [36].

To verify the effect of the AuNP layer on the impedance of the capsules, the local electrical conductivity of microcapsules (PSS/PAH) $)_{2}-A u N P S-(P S S / P A H)_{2}$ and $(\mathrm{PSS} / \mathrm{PAH})_{4}$ was investigated using a conductive AFM (C-AFM). A dc bias voltage of $1 \mathrm{~V}$ was applied between the AFM tip and the silicon substrate. For $(P S S / P A H)_{2^{-}}$ AuNPs-(PSS/PAH)2, a clear image of the local current was obtained (Figure 7c), which is consistent with its topographical image. The local currents on the capsule were smaller than the monolayer background, indicating a lower conductivity. However, for capsule (PSS/PAH) 4 , no contrast of local currents was obtained (Figure 7e), which reveals a significantly lower impedance compared to (PSS/PAH) $)_{2}-A u N P S-$ $(\mathrm{PSS} / \mathrm{PAH})_{2}$, thereby confirming the results obtained with SPIM.

Generally, gold nanoparticles on an electrode surface can improve its conductivity by providing conducting channels that facilitate electron transfer $[37,38]$. However, in the case of microcapsules, where nanoparticles were discontinuously inserted into the polyelectrolyte scaffolds, the formation of complete electric channels was not possible. Hence, the interactions between AuNPs and polyelectrolytes (PSS and 
PAH) probably caused the increase in impedance. Since AuNPs were covered and separated by polyelectrolyte chains, these highly dispersed particles may block the pores that exist on the PSS/PAH shell leading to a more densely packed structure with lower water content. This is also in agreement with the increased stiffness of AuNP modified capsules reported by Bedard et al. [11], which is also attributed to denser structures of multilayers when AuNPs are incorporated between layers.

\section{Conclusions}

We have demonstrated that the modification of PAH/PSS polyelectrolyte microcapsules with gold nanoparticles leads to an increase in the impedance of the capsule wall, which by far exceeds the increase expected due to the greater thickness of AuNP modified capsule walls alone. The dielectric constant of AuNP modified capsules was determined to be 39 in contrast to the values of 50 and 60 reported by other authors for pure PAH/PSS capsules [13, 14]. It is assumed that the gold nanoparticles decrease the porosity of the capsules and displace some of the water normally present in the capsule walls. The increase in impedance is in good agreement with the enhanced stiffness of AuNP modified capsules reported previously [11].

SPIM was shown to be a very effective technique for imaging the local impedance of microcapsules with high spatial resolution allowing visualisation and quantitative impedance measurements of overlapping and folded regions of the collapsed capsules using a simple circuit model (see Figure 6). Using a femtosecond $1250 \mathrm{~nm}$ laser with SAM modified SOS, SPIM images of microcapsules containing AuNPs were obtained, which were consistent with the morphology observed in optical images. The adhesion of microcapsules on SAM modified SOS by electrostatic 
attractions caused a local impedance increase and thus a photocurrent decrease in inversion. Furthermore, the photocurrent-voltage curves on capsules showed a negative voltage shift after normalisation due to the positively charged PAH outer layer. In contrast, capsules without AuNPs showed no SPIM response at all, confirming that the impregnation with AuNPs can significantly increase the impedance of microcapsules. The impedance variation of capsules with and without AuNPs deduced from SPIM was also verified by C-AFM measurements.

This study demonstrates that through the incorporation of AuNPs into PMLCs a significant increase in capsule impedance, and consequently a decrease in permeability, is realised. This has implications in drug delivery wherein the lowering of capsule wall water content should result in reduced ion exchange between the loaded capsule and the surrounding matrix. Additionally, the high level of resolution of PMLC images observed by SPIM holds great promise and indicates that the technique may be used in the future for the observation of interactions on surfaces with higher accuracy than is currently possible with other techniques. Of particular relevance may be its use as a new biomedical technique to study cell-surface interactions in a range of pathological conditions.

\section{Acknowledgements}

The authors are grateful to the China Scholarship Council for funding, the EU for providing a Marie-Curie Intra-European Fellowship (FLUOLAPS, PIIF-GA-2013627265), NEXUS (UK) for XPS measurements, Dr Weizhi Liu for training in microcapsule preparation, Dr Huijuan Cheng for providing AuNPs and Dr Nadezda Tarakina for help with TEM measurements.

\section{References}


[1] L.J. De Cock, S. De Koker, B.G. De Geest, J. Grooten, C. Vervaet, J.P. Remon, G.B. Sukhorukov, M.N. Antipina, Polymeric Multilayer Capsules in Drug Delivery, Angewandte Chemie International Edition, 49 (2010) 6954-6973.

[2] A.G. Skirtach, A.M. Yashchenok, H. Mohwald, Encapsulation, release and applications of LbL polyelectrolyte multilayer capsules, Chemical Communications, 47 (2011) 12736-12746.

[3] G.L. Beretta, M. Folini, F. Cavalieri, Y. Yan, E. Fresch, S. Kaliappan, C. Hasenohrl, J.J. Richardson, S. Tinelli, A. Fery, F. Caruso, N. Zaffaroni, Unravelling "off-target" effects of redox-active polymers and polymer multilayered capsules in prostate cancer cells, Nanoscale, 7 (2015) 6261-6270.

[4] M.F. Bedard, D. Braun, G.B. Sukhorukov, A.G. Skirtach, Toward self-assembly of nanoparticles on polymeric microshells: Near-IR release and permeability, ACS Nano, 2 (2008) 1807-1816.

[5] L.L. del Mercato, M.M. Ferraro, F. Baldassarre, S. Mancarella, V. Greco, R. Rinaldi, S. Leporatti, Biological applications of LbL multilayer capsules: From drug delivery to sensing, Advances in Colloid and Interface Science, 207 (2014) 139-154. [6] W.F. Dong, G.B. Sukhorukov, H. Mohwald, Enhanced Raman imaging and optical spectra of gold nanoparticle doped microcapsules, Phys. Chem. Chem. Phys., 5 (2003) 3003-3012.

[7] P. Rivera Gil, L.L. del Mercato, P. del-Pino, A. Munoz-Javier, W.J. Parak, Nanoparticle-modified polyelectrolyte capsules, Nano Today, 3 (2008) 12-21.

[8] F. Caruso, Nanoengineering of particle surfaces, Advanced Materials, 13 (2001) 11-22.

[9] M.F. Bédard, B.G. De Geest, A.G. Skirtach, H. Möhwald, G.B. Sukhorukov, Polymeric microcapsules with light responsive properties for encapsulation and release, Advances in Colloid and Interface Science, 158 (2010) 2-14.

[10] B. Radt, T.A. Smith, F. Caruso, Optically Addressable Nanostructured Capsules, Advanced Materials, 16 (2004) 2184-2189.

[11] M.F. Bedard, A. Munoz-Javier, R. Mueller, P. del Pino, A. Fery, W.J. Parak, A.G. Skirtach, G.B. Sukhorukov, On the mechanical stability of polymeric microcontainers functionalized with nanoparticles, Soft Matter, 5 (2009) 148-155.

[12] C.Y. Jiang, S. Markutsya, H. Shulha, V.V. Tsukruk, Freely suspended gold nanoparticle arrays, Advanced Materials, 17 (2005) 1669-+.

[13] R. Georgieva, S. Moya, S. Leporatti, B. Neu, H. Baumler, C. Reichle, E. Donath, $\mathrm{H}$. Mohwald, Conductance and capacitance of polyelectrolyte and lipid-

polyelectrolyte composite capsules as measured by electrorotation, Langmuir, 16 (2000) 7075-7081.

[14] T. Sun, C. Bernabini, H. Morgan, Single-Colloidal Particle Impedance Spectroscopy: Complete Equivalent Circuit Analysis of Polyelectrolyte Microcapsules, Langmuir, 26 (2010) 3821-3828.

[15] J. Wang, Y. Zhou, M. Watkinson, J. Gautrot, S. Krause, High-sensitivity lightaddressable potentiometric sensors using silicon on sapphire functionalized with self-assembled organic monolayers, Sens. Actuator B-Chem., 209 (2015) 230-236. [16] J. Wang, F. Wu, M. Watkinson, J. Zhu, S. Krause, "Click" patterning of selfassembled monolayers on hydrogen-terminated silicon surfaces and their characterization using light-addressable potentiometric sensors, Langmuir, 31 (2015) 9646-9654.

[17] L. Chen, Y. Zhou, S. Jiang, J. Kunze, P. Schmuki, S. Krause, High resolution LAPS and SPIM, Electrochemistry Communications, 12 (2010) 758-760. 
[18] S. Krause, W. Moritz, H. Talabani, M. Xu, A. Sabot, G. Ensell, Scanning PhotoInduced Impedance Microscopy - Resolution studies and polymer characterization, Electrochim. Acta, 51 (2006) 1423-1430.

[19] S. Krause, H. Talabani, M. Xu, W. Moritz, J. Griffiths, Scanning photo-induced impedance microscopy - an impedance based imaging technique, Electrochim. Acta, 47 (2002) 2143-2148.

[20] M.J. Schöning, A. Poghossian, Bio FEDs (Field-Effect devices): State-of-the-art and new directions, Electroanalysis, 18 (2006) 1893-1900.

[21] C. Wu, T. Bronder, A. Poghossian, C.F. Werner, M. Baecker, M.J. Schöning, Label-free electrical detection of DNA with a multi-spot LAPS: First step towards light-addressable DNA chips, Phys. Status Solidi A-Appl. Mat., 211 (2014) 14231428.

[22] C. Wu, T. Bronder, A. Poghossian, C.F. Werner, M.J. Schöning, Label-free detection of DNA using a light-addressable potentiometric sensor modified with a positively charged polyelectrolyte layer, Nanoscale, 7 (2015) 6143-6150.

[23] H.-J. Chen, D. Wen, Ultrasonic-aided fabrication of gold nanofluids, Nanoscale research letters, 6 (2011) 1-8.

[24] S. Ciampi, P.K. Eggers, G. Le Saux, M. James, J.B. Harper, J.J. Gooding, Silicon (100) Electrodes Resistant to Oxidation in Aqueous Solutions: An Unexpected Benefit of Surface Acetylene Moieties, Langmuir, 25 (2009) 2530-2539. [25] S. Ciampi, T. Böcking, K.A. Kilian, M. James, J.B. Harper, J.J. Gooding, Functionalization of Acetylene-Terminated Monolayers on Si(100) Surfaces: A Click Chemistry Approach, Langmuir, 23 (2007) 9320-9329.

[26] W.-F. Dong, G.B. Sukhorukov, H. Möhwald, Enhanced Raman imaging and optical spectra of gold nanoparticle doped microcapsules, Physical Chemistry Chemical Physics, 5 (2003) 3003-3012.

[27] M. James, S. Ciampi, T.A. Darwish, T.L. Hanley, S.O. Sylvester, J.J. Gooding, Nanoscale water condensation on click-functionalized self-assembled monolayers, Langmuir, 27 (2011) 10753-10762.

[28] A. Ng, S. Ciampi, M. James, J.B. Harper, J.J. Gooding, Comparing the Reactivity of Alkynes and Alkenes on Silicon (100) Surfaces†, Langmuir, 25 (2009) 13934-13941.

[29] C.C.A. Ng, S. Ciampi, J.B. Harper, J.J. Gooding, Antifouling behaviour of silicon surfaces modified with self-assembled monolayers containing both ethylene glycol and charged moieties, Surface Science, 604 (2010) 1388-1394.

[30] T. Palacin, H.L. Khanh, B. Jousselme, P. Jegou, A. Filoramo, C. Ehli, D.M. Guldi, S. Campidelli, Efficient functionalization of carbon nanotubes with porphyrin dendrons via click chemistry, Journal of the American Chemical Society, 131 (2009) 15394-15402.

[31] S. Ciampi, J.J. Gooding, Direct electrochemistry of cytochrome c at modified Si (100) electrodes, Chemistry-A European Journal, 16 (2010) 5961-5968.

[32] K. Miyamoto, T. Wagner, T. Yoshinobu, S. Kanoh, M.J. Schöning, Phase-mode LAPS and its application to chemical imaging, Sens. Actuator B-Chem., 154 (2011) 28-32.

[33] T. Wagner, M.J. Schöning, Chapter 5 Light-addressable potentiometric sensors (LAPS): recent trends and applications, in: S. Alegret, A. Merkoçi (Eds.)

Comprehensive Analytical Chemistry, Elsevier2007, pp. 87-128.

[34] R. Georgieva, R. Dimova, G. Sukhorukov, G. Ibarz, H. Möhwald, Influence of different salts on micro-sized polyelectrolyte hollow capsules, Journal of Materials Chemistry, 15 (2005) 4301-4310. 
[35] A. Poghossian, M.H. Abouzar, M. Sakkari, T. Kassab, Y. Han, S. Ingebrandt, A. Offenhäusser, M.J. Schöning, Field-effect sensors for monitoring the layer-by-layer adsorption of charged macromolecules, Sensors and Actuators B: Chemical, 118 (2006) 163-170.

[36] S. Leporatti, A. Voigt, R. Mitlohner, G. Sukhorukov, E. Donath, H. Mohwald, Scanning force microscopy investigation of polyelectrolyte nano- and microcapsule wall texture, Langmuir, 16 (2000) 4059-4063.

[37] G. Liu, S.G. lyengar, J.J. Gooding, An Electrochemical Impedance Immunosensor Based on Gold Nanoparticle-Modified Electrodes for the Detection of HbA1c in Human Blood, Electroanalysis, 24 (2012) 1509-1516.

[38] F.M. El-Cheick, F.A. Rashwan, H.A. Mahmoud, M. El-Rouby, Gold nanoparticlemodified glassy carbon electrode for electrochemical investigation of aliphatic dicarboxylic acids in aqueous media, J Solid State Electrochem, 14 (2010) 1425-1443. 
Figure 1

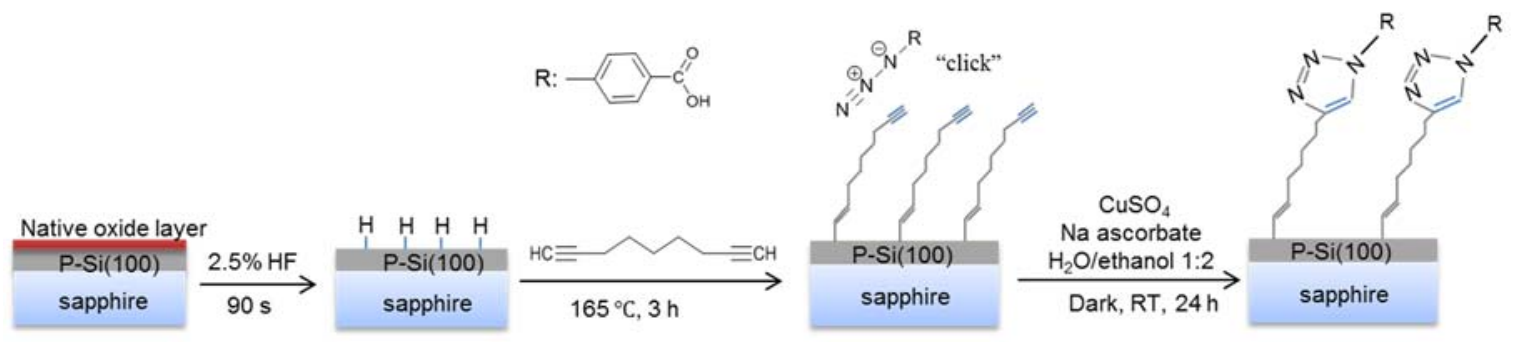

Figure 1 Modification of 1,8-nonadiyne monolayers on SOS substrates using "click" cycloaddition to generate $\mathrm{CO}_{2} \mathrm{H}$-terminated monolayers. 
Figure 2

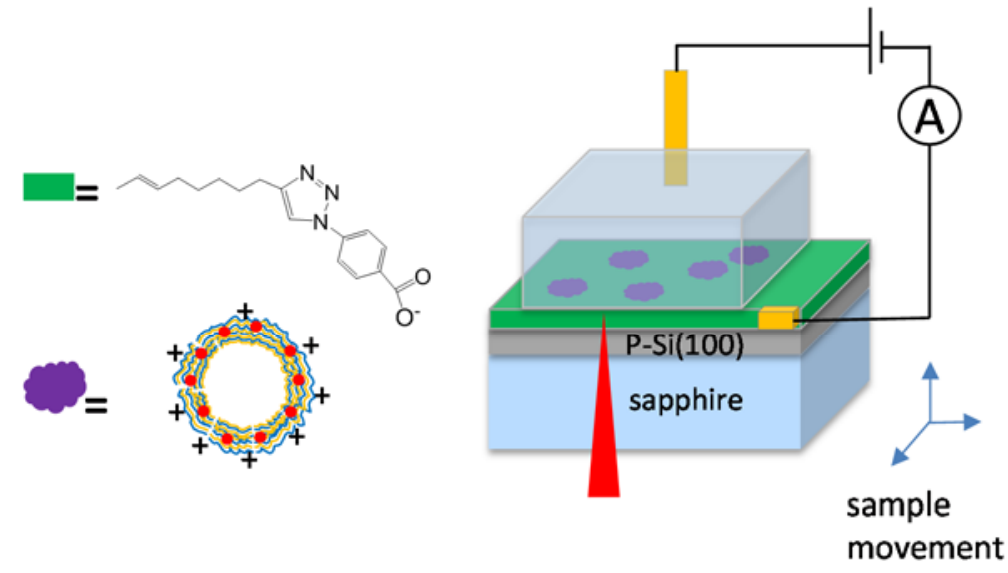

(a)

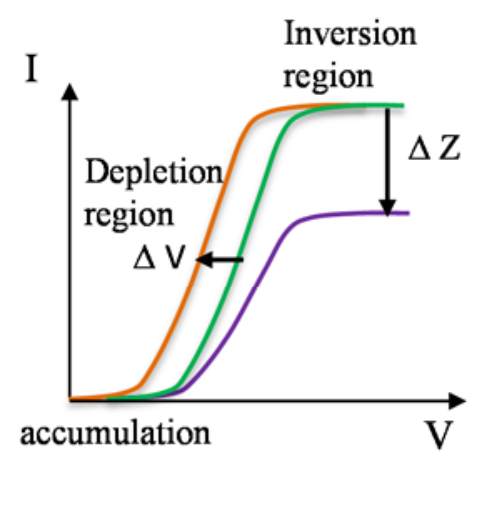

(b)

Figure 2 (a) Schematic drawing of the SPIM/LAPS principle with SAMs as the insulator for microcapsule imaging and (b) simulated photocurrent - voltage (I-V) curves for the corresponding SPIM/LAPS measurements; in SPIM, the variation (increase) in local impedance results in a change (decrease) of the maximum photocurrents in inversion; while for LAPS, surface potential changes (positive) shift the I-V curve along the voltage axis (negative) in depletion. 
Figure 3

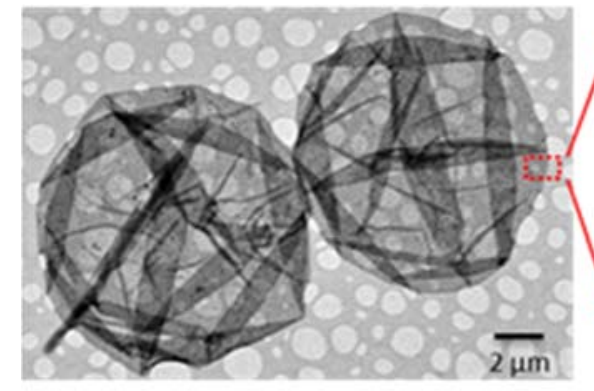

(a)

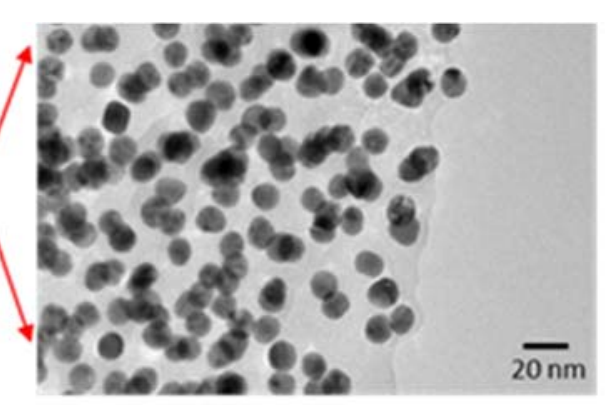

(b)

Figure 3 TEM images of two collapsed microcapsules labelled with AuNPs (a)

overview and (b) zoomed image clearly showing the even distribution of AuNPs in the shell of the capsule. 
Figure 4

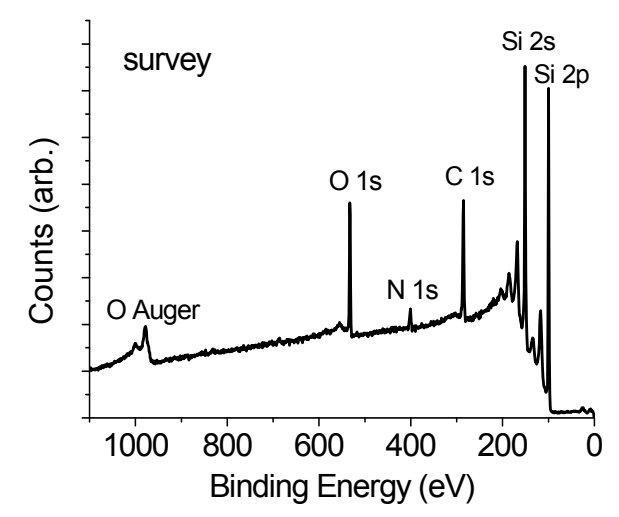

(a)

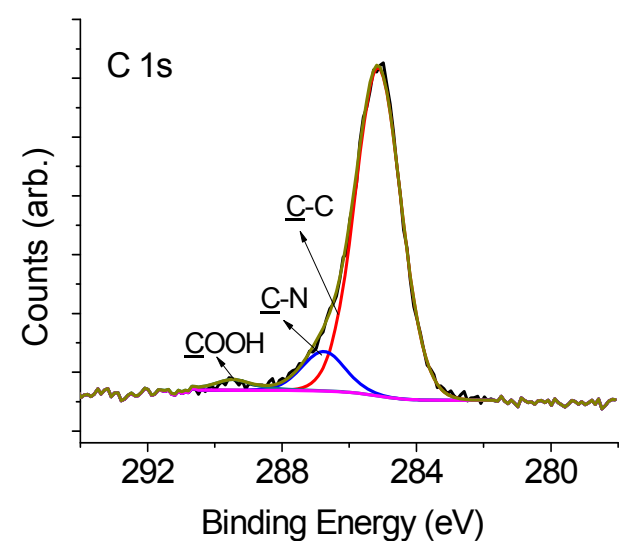

(c)

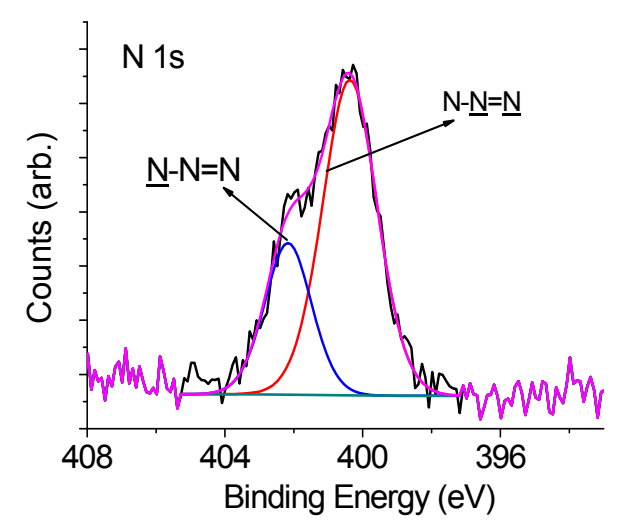

(b)

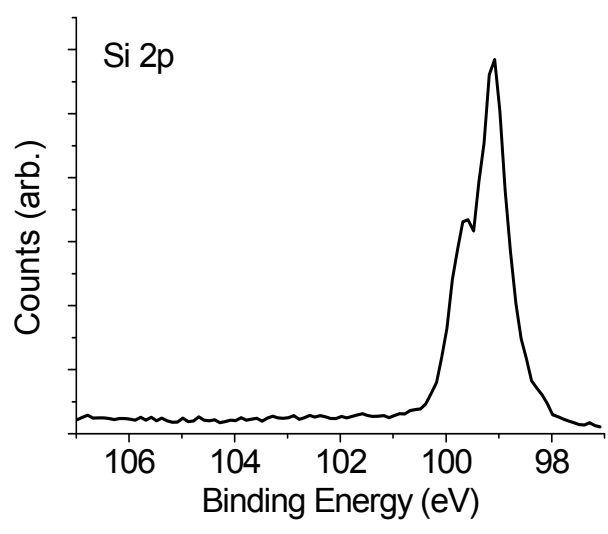

(d)

Figure 4 (a) XPS survey scan of 4-azidobenzoic acid functionalised SOS surface via "click" cycloaddition. High-resolution scans for (b) nitrogen, (c) carbon and (d) silicon. 
Figure 5

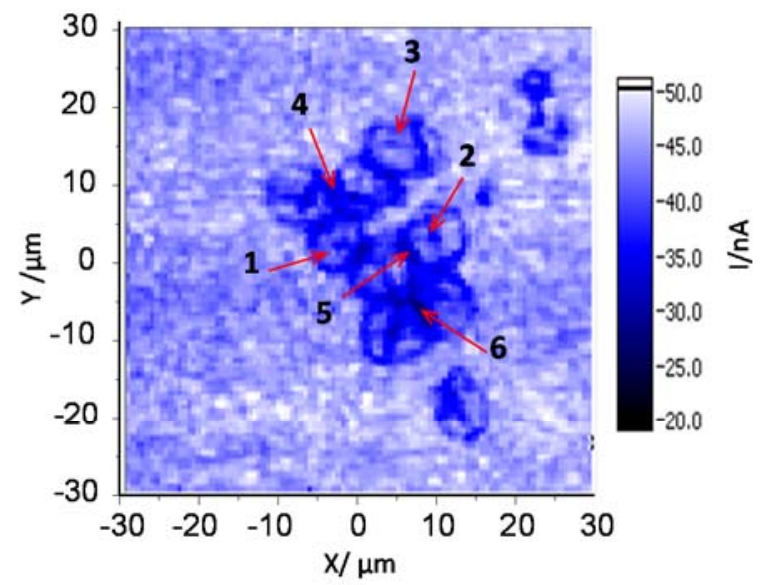

(a)

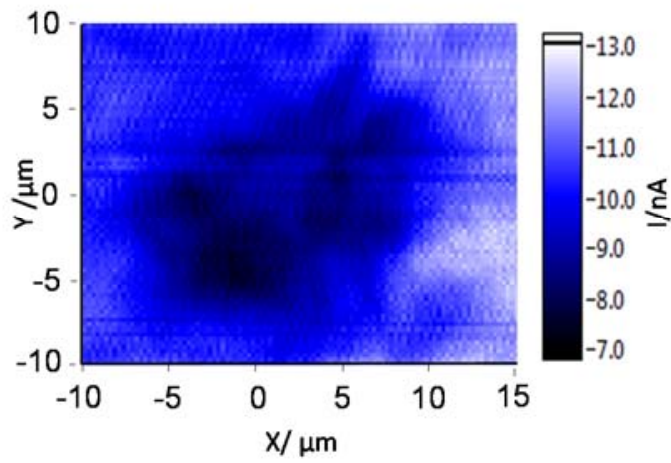

(c)

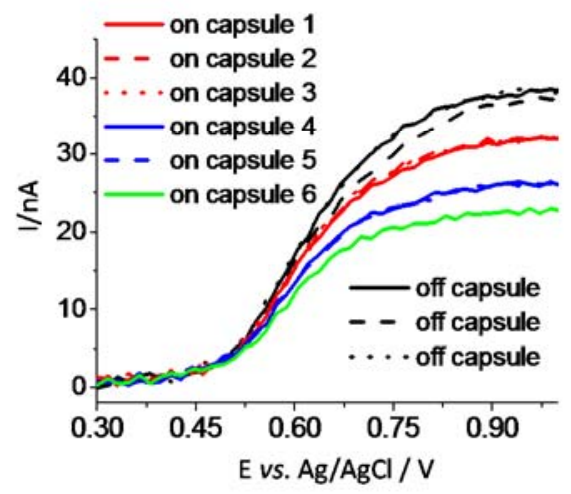

(e)

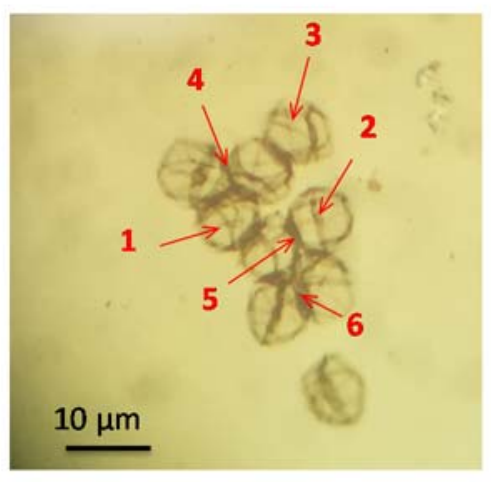

(b)

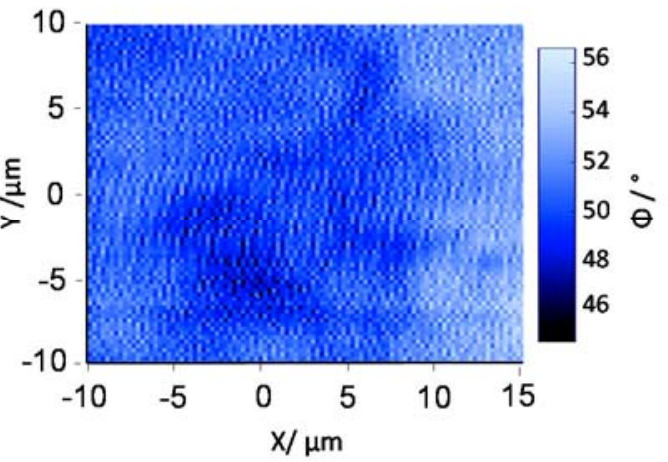

(d)

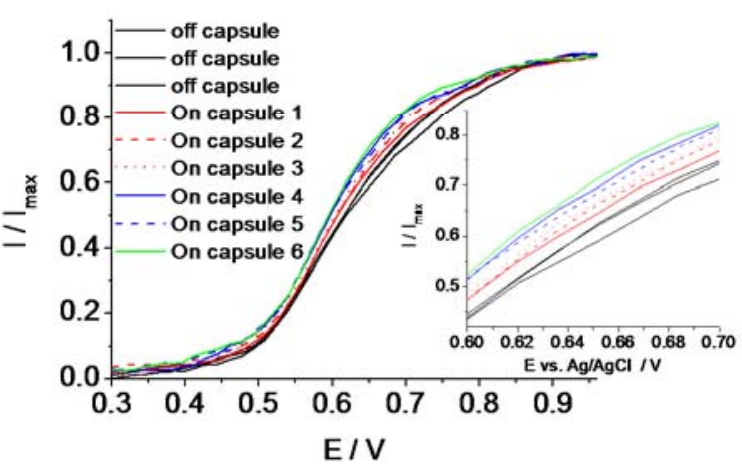

(f)

Figure $5(\mathrm{PSS} / \mathrm{PAH})_{2}$-AuNPs-(PSS/PAH $)_{2}$ attached on a COOH-terminated SOS substrate due to electrostatic attraction (a) SPIM image measured at $0.9 \mathrm{~V}$ (Vs. Ag/AgCl, scan range: $60 \mu \mathrm{m} \times 60 \mu \mathrm{m}$, step size: $0.6 \mu \mathrm{m}$, dwell time: $30 \mathrm{~ms}$ ) and (b) corresponding optical image; (c) SPIM image of single capsule scan range: $25 \mu m \times$ $20 \mu \mathrm{m}$, step size: $0.25 \mu \mathrm{m}$ and (d) corresponding phase angle image; (e) I-V curves on capsules and on monolayer modified surfaces and (f) normalised I-V curves. 
Figure 6

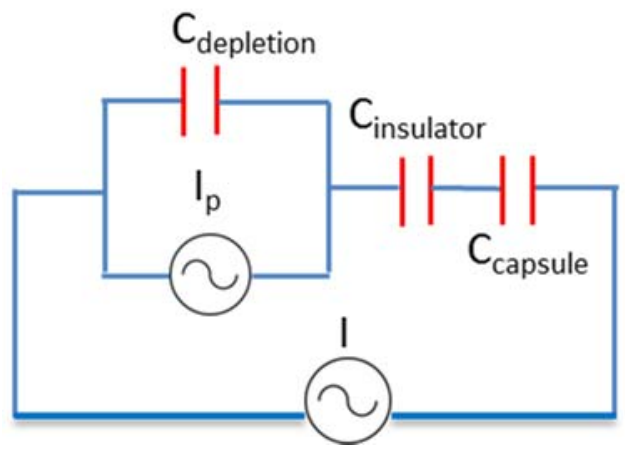

Figure 6 Equivalent circuit for photocurrent measurements with capsule. $I_{p}$ is the current photogenerated in the depletion layer and I is the measured photocurrent. 
Figure 7

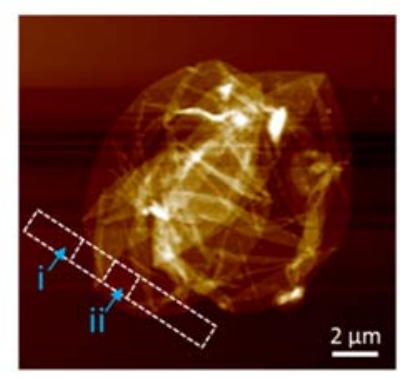

(a)

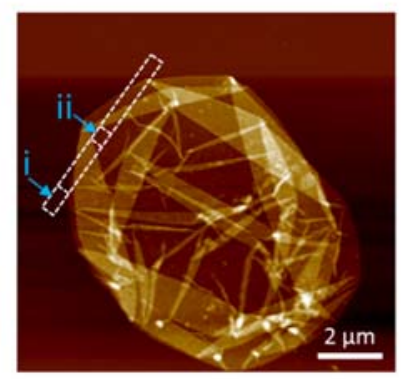

(d)

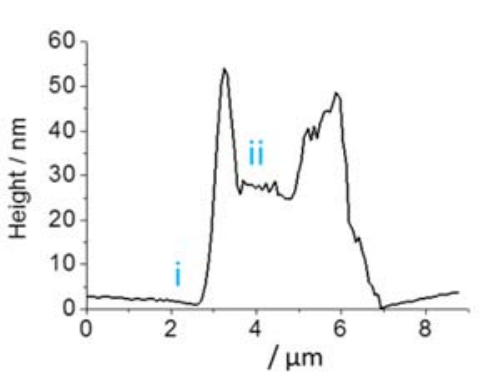

(b)

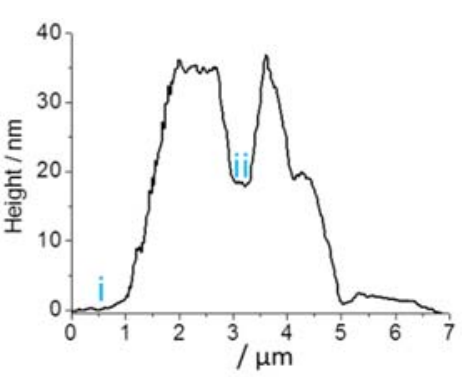

(e)

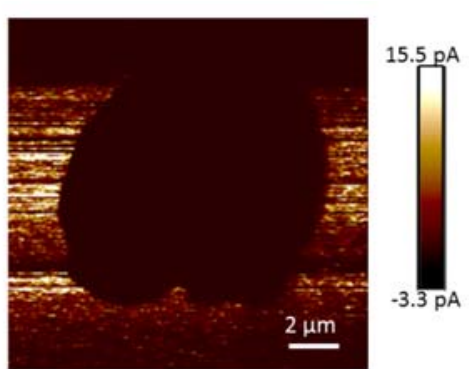

(c)

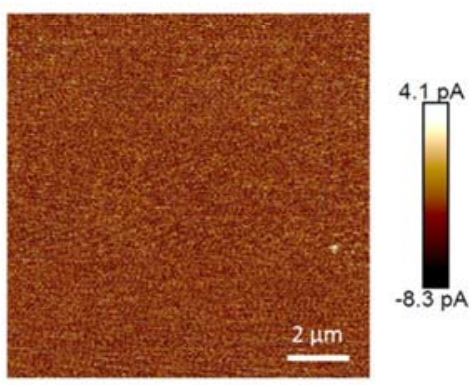

(f)

Figure 7 AFM results of microcapsule (PSS/PAH) $)_{2}-A u N P s-(P S S / P A H)_{2}$ (a)

topography image, (b) height profile along the rectangle indicated in (a) by the dotted white line and (c) corresponding C-AFM local current image, which shows lower currents on the capsule attached surface; microcapsules (PSS/PAH) 4 (d) topography image, (e) height profile along the rectangle indicated in (d) by the dotted white line and (f) corresponding C-AFM local current image showing no contrast between the background and capsule surfaces. 